\title{
Reducing Context-Bounded Concurrent Reachability to Sequential Reachability*
}

\author{
Salvatore La Torre ${ }^{1}$, P. Madhusudan ${ }^{2}$, and Gennaro Parlato ${ }^{1,2}$ \\ 1 Università degli Studi di Salerno, Italy \\ 2 University of Illinois at Urbana-Champaign, USA
}

\begin{abstract}
We give a translation from concurrent programs to sequential programs that reduces the context-bounded reachability problem in the concurrent program to a reachability problem in the sequential one. The translation has two salient features: (a) the sequential program tracks, at any time, the local state of only one thread (though it does track multiple copies of shared variables), and (b) all reachable states of the sequential program correspond to reachable states of the concurrent program.

We also implement our transformation in the setting of concurrent recursive programs with finite data domains, and show that the resulting sequential program can be model-checked efficiently using existing recursive sequential program reachability tools.
\end{abstract}

\section{Introduction}

Analysis of concurrent programs is an important problem that is difficult for a variety of reasons. The explosion in the number of interleavings between threads is one problem, and the explosion in the state-space needed to keep track of the combined states of each thread is another. For instance, even checking reachability of $n$ parallel systems, each modeled as a finite-state transition system (with some form of communication) is solvable only in time exponential in $n$, as there are exponentially many global configurations which are feasible.

In this paper, we consider the problem of translating concurrent programs to sequential programs, which reduces the reachability problem for the former to that for the latter. The motivation behind such a translation is to use the fairly sophisticated sequential analysis tools to analyze concurrent programs. For instance, this has been proposed in this same volume by Lahiri, Qadeer, and Rakamarić [5], in order to apply deductive verification tools based on SMT solvers to verify concurrent $\mathrm{C}$ programs.

A translation of the above kind is of course always possible - given a concurrent program, we can build a sequential program that simply simulates its (global) behavior. However, such a sequential interpreter would track the entire global state of the concurrent program, which involves keeping the local state of each thread. Our aim is to provide a translation that avoids this extreme blow-up

\footnotetext{
* This work was partially funded by NSF CAREER Award CCF 0747041, by the MIUR grants ex-60\% 2007-2008, and FARB 2009 Università degli Studi di Salerno (Italy).
} 
in state-space, and build a sequential program that tracks, at any point, the local state of only one thread of the concurrent program. However, such a translation is not always feasible.

A restricted reachability for concurrent programs has emerged in the last few years, mainly motivated from testing concurrent programs and model-checking finite-state models of them, called context-bounded reachability, wherein we ask whether an error state is reachable within $k$ context-switches, for a fixed $k$. This was first suggested by Qadeer and $\mathrm{Wu}$ [1], and has several appealing features.

First, it has been argued that bounded context-switching is a natural restriction as most concurrency related errors manifest themselves within a few contextswitches. Musuvathi and Qadeer have, for example, experimentally shown that a few context-switches explores a vast space of reachable configurations [9].

Second, when we deal with concurrent programs where variables range over finite domains (obtained either by restricting the domain or using some form of abstraction such as predicate abstraction), we obtain essentially concurrent pushdown systems, which have an undecidable reachability problem. However, it turns out, mainly due to results of [10], that the bounded context-switching reachability problem is decidable.

Finally, and perhaps most importantly, when one examines why contextbounded reachability is decidable for concurrent programs over finite data domains, the primary reason is that we can compositionally analyze the program, examining each thread separately and combining the results. This compositional reasoning hence involves searching a state-space where at any point only the local state of a single thread is tracked.

In [6], Lal and Reps propose a transformation of concurrent programs to sequential programs that reduces context-bounded reachability in the former to reachability in the latter. This translation exploits compositional arguments underlying the decidability proofs to construct a sequential program that tracks, at any point, the local state of one thread, the shared state, as well as $k$ copies of the shared state (corresponding to the shared state at the $k$ context-switches). This translation is appealing when the local state is complex (in particular, when the local state has a stack to model recursive control), and the shared state is comparably less complex.

The translation proposed by Lal and Reps, however, does not permit a lazy analysis: the sequential program guesses in advance the valuations of shared variables $g_{1}, \ldots, g_{k}$ at the context-switches, and verifies each thread locally against this guess. Hence, the sequential program unnecessarily explores unreachable states of the concurrent program (as the guessed $g_{i}$ 's may not be reachable).

In our opinion, a transformation that results in a lazy analysis (one which explores only reachable states) is highly desirable; for example, in model-checking, it can drastically reduce the size of the state-space that needs to be explored. In fact, Lal and Reps [6] do give direct lazy analysis algorithms for finite-state programs, and our recent work in [4] also provides a direct fixed-point algorithm for lazy analysis. However, a transformation from concurrent programs to sequential programs that preserves laziness was not known. 
Contributions. In this paper, we show a lazy translation: given a concurrent program and a bound $k$, we show how it can be transformed to a sequential program, such that reachability within $k$ context-switches in the concurrent program reduces to reachability in the sequential one. Moreover, the salient feature of the translation is that the reachable states of the sequential program correspond to reachable states of the concurrent program, and hence is, in our opinion, a more faithful representation of the sequential program. The main idea behind our reduction is to have the sequential program calling individual threads multiple times from scratch in order to recompute the local states at context-switches.

We also implement an eager translation and our lazy translation for concurrent programs over finite data-domains (a.k.a. concurrent Boolean programs). This results in sequential programs over a finite data-domain, which can be modelchecked using existing tools. Our implementations of the translations are available online 1 . We show that our laziness-preserving transformation outperforms eager transformations on a class of multithreaded Bluetooth driver examples (the original programs and the transformed ones are also available online).

The paper is organized as follows: Section 2 gives a high-level and intuitive description of our eager and lazy translations; Section 3 formally defines the class of sequential and concurrent programs; Section 4 describes the eager translation (which is mainly adapted from ideas in [6]); the laziness-preserving translation is presented in Section 5; Section 6 reports on our implementation and experiments for Boolean programs; and Section 7 concludes with some future directions.

Related Work. Bounded context-switching reachability was introduced in [1]: the KISS project implemented reachability within two context-switches and found data-race errors in device drivers; interestingly, it also reduced the problem to sequential reachability (the reduction is simple for two switches). Decidability of context-bounded analysis for concurrent recursive Boolean programs was established in [10] using automata theoretic methods. There have been a number of analysis algorithms and implementations of context-bounded reachability problems: [13] implement the automata-theoretic solution symbolically, 6] propose an algorithm to compute the reachable states lazily, and the work in [4] implements symbolic fixed-point based solutions for lazy reachability. Bounded context-switching has also been exploited in other contexts: the tool CHESS [8] explores bounded context-switching interleavings to test concurrent programs, and bounded context-switching for systems with heaps [1], systems communicating using queues [3], and weighted pushdown systems [7] have been proposed.

Bounded context-switches vs bounded rounds. Lal and Reps [6], apart from giving a transformation for eager analysis, also make a technical improvement: by using only $k$ extra sets of shared variables, we can explore all the state space reachable in $k$ round-robin rounds (which is larger than that reached in $k$ context-switches).

In both our transformations, we have considered bounded context-switching reachability, and not bounded round-robin reachability (this is why our eager transformation is slightly different from that of [6] $)$. The reason is that we see no

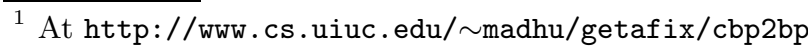


efficient way of transforming programs using only $O(k)$ sets of global variables that effects a lazy-transformation. While we do know of such a translation, this requires calling each thread too many times (exponentially) many times, which we believe will not work well in practice. This translation is out of the scope of this paper, and the problem of coming up with a more efficient translation for bounded rounds reachability is an open problem.

\section{From Concurrent to Sequential Programs}

In this section, we briefly sketch two translation schemes from concurrent to sequential programs which preserve reachability up to a fixed number of contextswitches. The transformations work for general programs (even when the domain of variables is not bounded), except that they assume that the state of shared variables is known, and can be replicated and compared against each other.

Of course, there is a simple translation of concurrent programs to sequential programs that keeps the entire global configuration of the program. However, our aim here is to build a sequential program that keeps only the local state of a single thread at any time (though we allow some copies of shared states). In particular, when applied to concurrent recursive programs over finite datadomains, the translation will should yield a sequential recursive program over a finite data-domain; this would reduce concurrent context-bounded reachability on finite domains to reachability of sequential programs over finite domains, which is a decidable problem.

We consider concurrent programs with a fixed number of threads that communicate with each other using shared variables. Each thread is a sequential program (with possibly recursive procedures). A state is thus given by the values of the shared variables and the local state of each thread. A computation of a concurrent program is a sequence of contexts, where in each context a single thread has control.

For ease of presentation let us first consider the case of a concurrent program with only two threads $T_{1}$ and $T_{2}$. Also, instead of describing the transformation, we take one particular path in the concurrent program, and describe how the corresponding sequential program will discover it.

Consider a sample computation whose control flow at the thread level is shown on the right. It starts in a state $s_{0}$ with the control in $T_{1}$, then at a state $s_{1}$ switches the control to $T_{2}$ at state $s_{2}$, then locally executes $T_{2}$ till it reaches the state $s_{3}$, and then switches to $T_{1}$ again at state $s_{4}$, and so on. In a simple sequential simulation of this computation, while we execute instructions of $T_{2}$, say from $s_{2}$, we need to

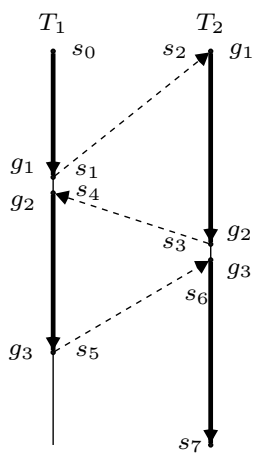
remember the local component of thread $T_{1}$ in state $s_{1}$, in order to compute the switch from $s_{3}$ to $s_{4}$. This can be very expensive. In the case of finite data domains, where threads have recursive procedures and we would like to build a sequential program also over a finite data domain, this is in fact impossible, as it 
involves keeping the unbounded call-stack of $T_{1}$ at $s_{1}$. Schemes for translation to sequential programs hence need to explore this run in a different manner, where only the local state of one process is kept at any given point.

The two translation schemes we present in the rest of this section do not store the local states of more than one thread, but will store $k$ valuations of shared variables, which intuitively correspond to the value of shared variables at context-switches. In the computation sketched above, if $g$ is the set of shared variables, we denote by $g_{1}$ the value of the shared variables at the first contextswitch from $T_{1}$ to $T_{2}$, i.e., the value of shared variables at states $s_{1}$ and $s_{2}$. Similarly, let $g_{2}$ be the value at the next context-switch back to $T_{1}$ (i.e., at states $s_{3}$ and $s_{4}$ ), and $g_{3}$ the value at the last context-switch back to $T_{2}$ (i.e., at states $s_{5}$ and $s_{6}$ ).

The eager and lazy translations differ in the way they compute the values of these shared variables at the context-switches: the eager approach nondeterministically guesses them right at the beginning, while the lazy approach computes them dynamically.

The eager approach. In the eager approach, we guess the values $g_{1}, g_{2}, g_{3}$ at the beginning (non-deterministically). Then, we process thread $T_{1}$ completely, handling both segments of its run $\left(s_{0}\right.$ to $s_{1}$ and $s_{4}$ to $\left.s_{5}\right)$, and then erase the local state and proceed to process the two segments of thread $T_{2}\left(s_{2}\right.$ to $s_{3}$ and $s_{6}$ to $\left.s_{7}\right)$. Note that each thread is processed only once.

More precisely, after guessing $g_{1}, g_{2}$ and $g_{3}$, we start to compute in $T_{1}$ the first context. At any point where the shared variables match the guessed value $g_{1}$, we allow a "jump" where the global variables are rewritten to $g_{2}$, and we proceed in $T_{1}$ computing the third context, till we reach $g_{3}$. Note that across the "jump" above (from $s_{1}$ to $s_{4}$ ), the local state of thread $T_{1}$ is preserved. Hence the call to thread $T_{1}$ verifies that there is a run which can reach shared-variable-state $g_{1}$, jump to $g_{2}$, and proceed to reach $g_{3}$.

Next, we erase the local state of $T_{1}$ and proceed to process thread $T_{2}$ from its initial local states with the shared variables assigned with $g_{1}$. We then continue till we reach a state with shared-variable valuation $g_{2}$, jump to $g_{3}$ preserving local state, and continue the computation of the fourth context in $T_{2}$.

Intuitively, the guessed values $g_{1}, g_{2}, g_{3}$ allow the stitching of the two execution segments that have been executed in the two threads. However, since the tuple of values of the shared variables is guessed, they may not correspond to reachable states of the concurrent program, and hence may lead to exploring the individual threads on unreachable regions of the state-space.

The lazy approach. In the lazy approach, we start computing $T_{1}$ in the first context from an initial global state. At any point of the execution, we can choose nondeterministically to switch context, in which case we store the value of the shared variables in $g_{1}$ and terminate the thread (thereby losing its local state).

Then, we execute $T_{2}$ in the second context, starting from a local initial state and shared variables initialized with $g_{1}$. At any point of the execution, we can choose nondeterministically to switch context, in which case we store in $g_{2}$ the value of the shared variables and terminate the thread (again losing the local state). 
We then would like to start executing $T_{1}$ (i.e., from $s_{4}$ ) in the third context, but since we had lost its local state, we need to recompute it. Thus, starting from an initial global state, we execute $T_{1}$ until we reach a state matching $g_{1}$ on the shared variables. (Notice that this may be an entirely different local state than the one we explored in the first context! However, as we show, this does not affect correctness.)

At such a point, we allow the thread to replace the value of the shared variables with $g_{2}$, and proceed to execute $T_{1}$ to compute the third context (from $s_{4}$ to $\left.s_{5}\right)$. Again, at any point, we can decide nondeterministically to switch context, in which case we store the value of the shared variables in $g_{3}$, and end thread $T_{1}$.

Finally, we move to execute $T_{2}$ in the fourth context. As we $\operatorname{did}$ for $T_{1}$, we need to restart thread $T_{2}$ from its initial state to recompute the local state at the beginning of the fourth context (i.e. at $s_{3}$ or $s_{6}$ ). Thus, we simulate $T_{2}$ with shared variables initialized to $g_{1}$, wait for it to reach a state with shared variables matching $g_{2}$, non-deterministically choose to assign the global variables with $g_{3}$, and proceed to compute the fourth context (from $s_{6}$ to $s_{7}$ ). Again, the local state produced on this new invocation of thread $T_{2}$ may be entirely different, and yet the states discovered in the fourth context are indeed reachable.

In contrast to the eager approach, the values of $g_{1}, g_{2}, g_{3}$ are computed dynamically, and thus are guaranteed to be reachable by the concurrent program. This can be sometimes a huge advantage as large (and complex) portions of the state space are spared from analysis. More importantly, we believe that this is a more faithful representation of the concurrent program. On the other hand, it is true that this approach executes each context several times (at most $k / 2$ times).

Generalization to multiple threads. Consider now a concurrent program with $n$ threads $T_{1}, \ldots, T_{n}$ and a fixed integer $k>0$. Let the variables $g_{i}(i=$ $1, \ldots, k)$ hold the valuation of the shared variables at the $i$-th context-switch, and let the variables $t_{i}(i=0, \ldots, k)$ hold the index of the thread that has control in the $(i+1)$-th context.

In the eager approach, we start guessing non-deterministically both tuples $g_{1}, \ldots, g_{k}$ and $t_{0}, \ldots, t_{k}$. Then, we run each thread $T_{i}$, for $i=1, \ldots, n$, through all the contexts where it has the control (i.e., all contexts $j$ such that $t_{j}=i$ ) and in doing this we check that the context-switches happen at states that match the values of the shared variables in the tuple $g_{1}, \ldots, g_{k}$.

In the lazy approach, the thread scheduling $t_{0}, \ldots, t_{k}$ is determined nondeterministically as and when the context-switches happen, and each $g_{i}$ is computed by executing the thread $T_{j}$ which has the control in the context $i$ (i.e., such that $j=t_{i}$ ) through all the contexts in which it had control up until context $i$. As in the previous approach, when re-executing the thread in the previously computed contexts, we check that the context-switches happen at states that match the values of the shared variables in the already computed tuple $g_{1}, \ldots, g_{i-1}$.

\section{Concurrent Programs}

Sequential recursive programs. Let us fix the syntax of a simple sequential programming language with variables ranging over only the integer and Boolean 
domains, and with explicit syntax for nondeterminism, (recursive) function calls, and tuples of return values.

The transformations in this paper require that we can cache the shared variables, and copy and compare them, and the notation is far simpler when we do not have complex or dynamically allocated structures. Handling domains of types other than integers is straightforward, but we will stick to integers for simplicity.

Programs are described by the following grammar:

$$
\begin{aligned}
& \langle p g m\rangle \quad::=\langle\text { gvar-decl }\rangle ;\langle\text { proc-list }\rangle \\
& \langle\text { gvar-decl }\rangle::=\text { decl int } x \mid \text { decl bool } x \mid\langle\text { gvar-decl }\rangle ;\langle\text { gvar-decl }\rangle \\
& \langle\text { proc-list }\rangle \quad::=\langle\text { proc }\rangle\langle\text { proc-list }\rangle \mid\langle\text { proc }\rangle \\
& \langle\text { proc }\rangle \quad::=f^{h, m}\left(x_{1}, \ldots, x_{h}\right) \text { begin }\langle\text { lvar-decl }\rangle ;\langle\text { stmt }\rangle \text { end } \\
& \langle\text { lvar-decl }\rangle::=\operatorname{decl} \text { int } x \mid \operatorname{decl} \text { bool } x \mid\langle\text { lvar-decl }\rangle \text {; }\langle\text { lvar-decl }\rangle \\
& \langle\text { stmt }\rangle \quad::=\langle\text { stmt }\rangle ;\langle\text { stmt }\rangle|\operatorname{skip}|\langle a s s i g n\rangle \mid \text { assume }(\langle b \text {-expr }\rangle) \mid \\
& \text { call } f^{h, 0}\left(x_{1}, \ldots, x_{h}\right) \quad \mid \text { return } x_{1}, \ldots, x_{m} \mid \\
& \text { if }(\langle b \text {-expr }\rangle) \text { then }\langle\text { stmt }\rangle \text { else }\langle s t m t\rangle \text { fi } \mid \\
& \text { while }(\langle b-e x p r\rangle) \text { do }\langle s t m t\rangle \text { od } \\
& \langle\text { assign }\rangle \quad::=x_{1}, \ldots, x_{m}:=\langle\exp r\rangle_{1}, \ldots,\langle\exp r\rangle_{m} \mid \\
& x_{1}, \ldots, x_{m}:=f^{h, m}\left(y_{1}, \ldots, y_{h}\right) \\
& \langle\text { expr }\rangle \quad::=x|c|\langle b-\exp r\rangle \\
& \langle b \text {-expr }\rangle \quad::=T|F| *|x| \neg\langle b \text {-expr }\rangle \mid\langle b \text {-expr }\rangle \vee\langle b \text {-expr }\rangle
\end{aligned}
$$

In the above, $x, x_{i}, y_{i}$ are from a set of variable names $\operatorname{Var}, c$ is any integer constant, and $f^{h, m}$ denotes a function with $h$ formal parameters and $m$ return values. Some of the functions $f^{h, m}$ may be interpreted to have existing functionality, such as integer addition or library functions, in which case their code is not given and we assume they happen atomically.

A program has a global variable declaration followed by a list of functions. Each function is a declaration of local variables followed by a sequence of statements, where statements can be tuple assignments, calls to functions (call-byvalue) that take in multiple parameters and return multiple values, conditional statements, while-loops, or return statements. Expressions can be integer constants, variables or Boolean expressions. Boolean expressions can be true, false, or non-deterministically true or false $(*)$, and can be combined using standard Boolean operations. Functions that do not return any values are called using the call statement. We also assume that the program type-checks with respect to the integer and Boolean types.

We will assume several obvious restrictions on the above syntax: global variables and local variables are assumed to be disjoint; formal parameters are local variables; the body of a function $f^{h, m}$ has only variables that are either globally declared, locally declared, or a formal parameter; a return statement in the body of $f^{h, m}$ is of the form return $x_{1}, \ldots, x_{m}$ or simply return (in the latter case arbitrary values will be returned).

Let us also assume that there is a function main, which is the function where the program starts, and that there are no calls to this function in the code of $P$. 
The semantics is the obvious one: a configuration of a program consists of a stack which stores the history of positions at which calls were made, along with valuations for local variables, and the top of the stack contains the local and global valuations, and a pointer to the current statement being executed.

The reachability problem asks whether a particular statement in the program marked using a special label Target is reachable.

Concurrent programs. A concurrent program is a finite set of recursive programs running in parallel and sharing some (global) variables.

Formally, the syntax of concurrent programs is defined by extending the syntax of sequential recursive programs with the following rules:

$$
\begin{array}{ll}
\langle\text { conc-pgm }\rangle & :=\langle\text { svar-decl }\rangle ;\langle\text { init }\rangle\langle\text { pgm-list }\rangle \\
\langle\text { svar-decl }\rangle & :=\text { decl int } x \mid \text { decl bool } x \mid\langle\text { svar-decl }\rangle ;\langle\text { svar-decl }\rangle \\
\langle\text { init }\rangle & ::=\langle\text { proc }\rangle \\
\langle\text { pgm-list }\rangle & ::=\langle\text { pgm }\rangle\langle\text { pgm-list }\rangle \mid\langle\text { pgm }\rangle
\end{array}
$$

Let $\mathcal{P}$ be a concurrent program formed by the sequential programs $P_{1}, \ldots, P_{n}$ (where $n>0$ ). Each program $P_{i}$ has its own global and local variables, and also has access to variables that are shared with the other component programs. Let us assume that each concurrent program has a function init where the shared variables are initialized (corresponding to the init construct in the above abstract syntax). Let us further assume that the function main of each component program $P_{i}$ is named thread . $_{\text {. }}$

The behavioral semantics of $\mathcal{P}$ is obtained by interleaving the behaviors of $P_{1}, \ldots, P_{n}$. At the beginning of any computation the shared variables are set according to function init. At any point of a computation, only one of the programs is active. Therefore, a state of $\mathcal{P}$ is denoted by a tuple $\left(i, u_{S}, \bar{u}_{1}, \ldots, \bar{u}_{n}\right)$ where $P_{i}$ is the currently active program, $u_{S}$ is a valuation of the shared variables and $\bar{u}_{j}$ is a state of $P_{j}$ for $j=1, \ldots, n$. From such a state the computation of $\mathcal{P}$ can evolve either according to the local behavior of $P_{i}$ or by switching to another program $P_{j}$, which then becomes the new active program. A maximal consecutive part of a computation visiting only states where the same program $P_{i}$ is active is called a context.

The reachability problem for concurrent programs asks whether a particular statement in the program marked using a special label Target is reachable. The reachability problem for concurrent programs under a context-switch bound $k$, for $k \geq 1$, asks whether Target is reachable within $k$ context-switches.

Example 1. Figure 1 illustrates a simple concurrent program with two component programs starting at thread t $_{1}$ and thread $_{2}$ respectively, and five shared variables test, $x_{1}, \ldots, x_{4}$, all over the Boolean domain. Function init assigns the initial value to the shared variable test and leaves all the others unassigned.

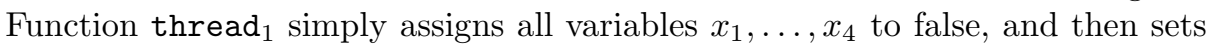

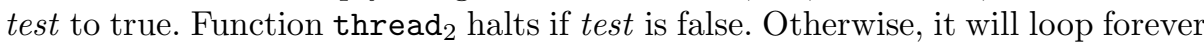
by nondeterministically choosing either to swap $x_{1}$ and $x_{2}$, or to shift the bits $x_{1}, \ldots, x_{4}$ circularly. The instruction labeled with Target is never reached on 


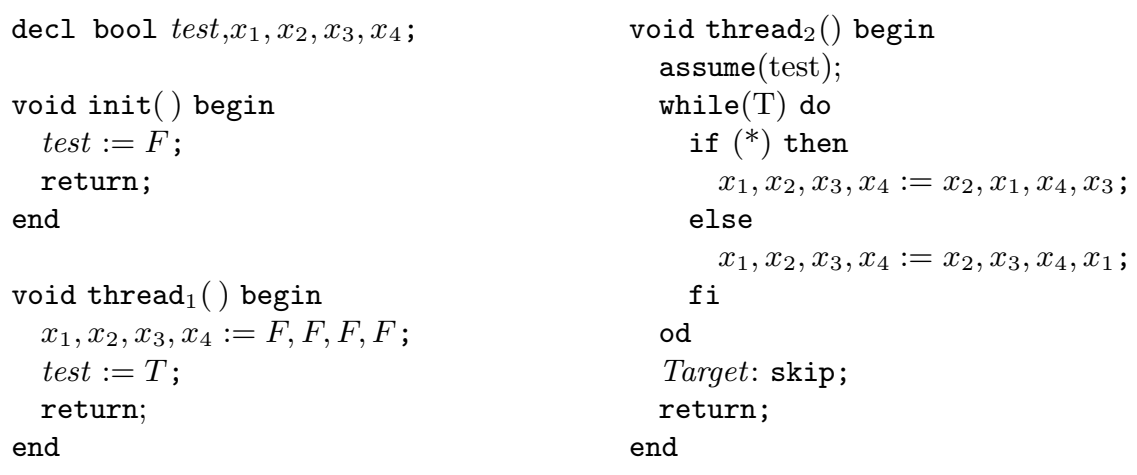

Fig. 1. The concurrent program permutation

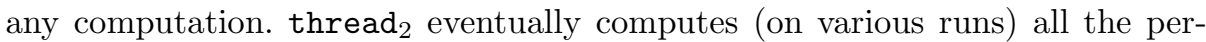
mutations of the bits stored in $x_{1}, \ldots, x_{4}$. Note that, since these variables are

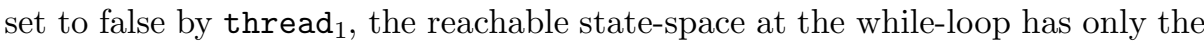
valuation $\left\langle x_{1}=F, x_{2}=F, x_{3}=F, x_{4}=F\right\rangle$.

\section{Translation Scheme: The Eager Approach}

In this section, we give a detailed description of the translation of a concurrent program $\mathcal{P}$ with target program counter $p c$ under a context-switching bound $k$ to a sequential program using the eager approach (denoted $\operatorname{Eager}_{k}(\mathcal{P}, p c)$ ), and argue its correctness.

Besides the variables in $\mathcal{P}$, the sequential program $\operatorname{Eager}_{k}(\mathcal{P}, p c)$ will have extra global variable tuples $g_{1}, \ldots, g_{k}$ and $t_{0}, \ldots, t_{k}$, as described in Section 2 We add also the following control variables: a variable $t$ to keep the index of the active component program, a variable $c x$ to keep the current context number, a Boolean variable terminate to interrupt the execution of a program component, and a Boolean variable goal which gets set to true when the target $p c$ of $\mathcal{P}$ is reached.

The sequential program $\operatorname{Eager}_{k}(\mathcal{P}, p c)$ is composed of: two new functions main and contextSwitch that are shown in Figure 2, and for every function $P$ of $\mathcal{P}$, a function $P^{e}$ which is a transformation of $P$.

In Figure 2, we use nextContext $\left(c x, t, t_{0}, \ldots, t_{k}\right)$ to denote a function that computes the index of the first context in which $P_{t}$ is active after $c x$, if any, and $k+1$, otherwise. Formally, nextContext $\left(c x, t, t_{0}, \ldots, t_{k}\right)$ is the value $i$ such that either $t_{i}=t$ and $t_{j} \neq t$ for all $j$ s.t. $c x<j<i$, or $i=k+1$ and $t_{j} \neq t$ for all $j>c x$. Clearly, such an index can be computed with a few lines of code. We also use firstContext $\left(t, t_{0}, \ldots, t_{k}\right)$ to compute the first context in which $P_{t}$ is active in the computation. 
Let $g$ : shared variables of $\mathcal{P}$; Let $g_{1}, \ldots, g_{k}$ be $k$ copies of $g$; decl int $c x, t, t_{0}, \ldots, t_{k} ; \quad$ decl bool goal, terminate;

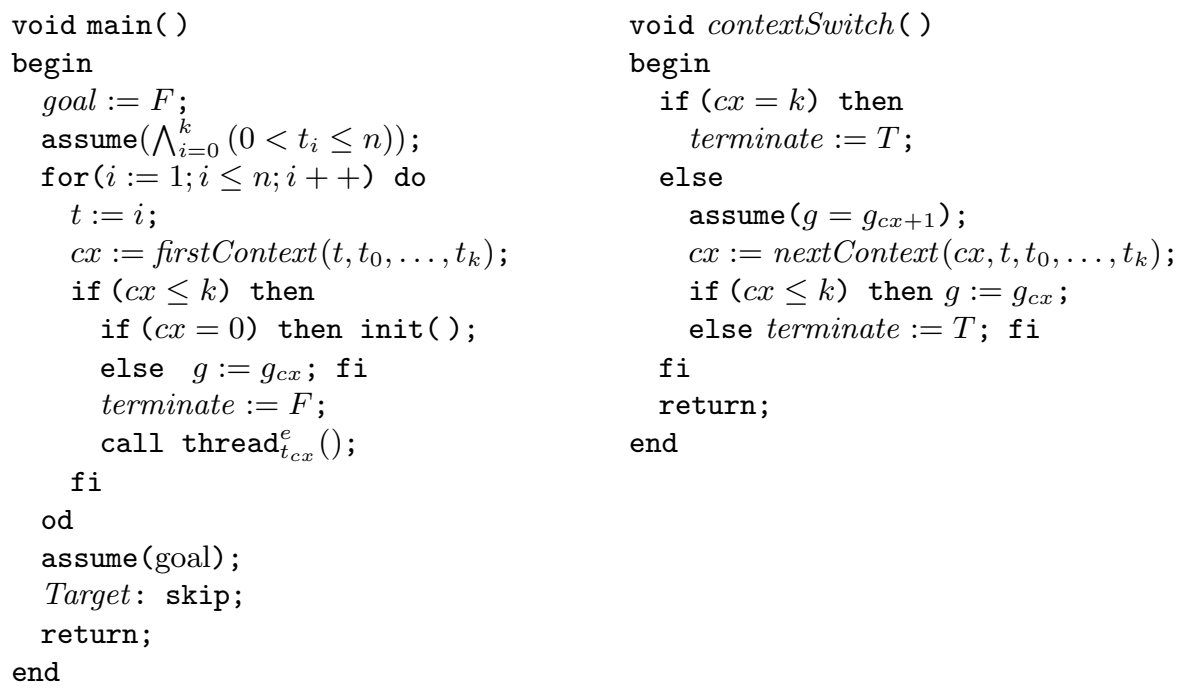

Fig. 2. Functions main and contextSwitch of the program $\operatorname{Eager}_{k}(\mathcal{P}, p c)$

Each function $P^{e}$ is obtained from the corresponding function $P$ of $\mathcal{P}$ by a simple transformation. We first interleave the statements of $P$ with the lines of control code $\mathcal{C}$ shown in Figure 3 . More precisely, we rewrite the

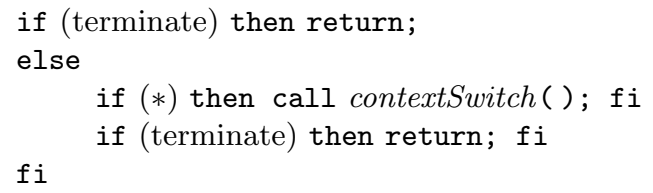

Fig. 3. Control code statements of $P$ according to the following rules $(\mathcal{E}$ is an arbitrary Boolean expression, $\mathcal{S}_{1}$ and $\mathcal{S}_{2}$ are arbitrary statements, and $\mathcal{S}$ is a basic statement of the kind assign or skip or assume or call):

$$
\begin{aligned}
& -\tau\left[\mathcal{S}_{1} ; \mathcal{S}_{2}\right]=\tau\left[\mathcal{S}_{1}\right] ; \tau\left[\mathcal{S}_{2}\right] \\
& -\tau[\mathcal{S}]=\mathcal{S} ; \mathcal{C} \\
& -\tau\left[\text { return } x_{1}, \ldots, x_{m}\right]=\operatorname{return} x_{1}, \ldots, x_{m} \\
& -\tau\left[\text { while }(\mathcal{E}) \text { do } \mathcal{S}_{1} \text { od }\right]=\text { while }(\mathcal{E}) \text { do } \mathcal{C} ; \tau\left[\mathcal{S}_{1}\right] \text { od } \\
& -\tau\left[\text { if }(\mathcal{E}) \text { then } \mathcal{S}_{1} \text { else } \mathcal{S}_{2} \text { fi }\right]=\text { if }(\mathcal{E}) \text { then } \mathcal{C} ; \tau\left[\mathcal{S}_{1}\right] \text { else } \mathcal{C} ; \tau\left[\mathcal{S}_{2}\right] \text { fi }
\end{aligned}
$$

Actually, we can optimize the above translation by inserting control code only after statements that read or write a shared variable, or calls to a function.

Next, we insert "assume(F);" before each return statement of the functions thread $_{i}$ for $i=1, \ldots, n$; this prevents thread $_{i}$ from returning to main after executing to completion. Finally, we insert "goal:=T;" right before the statement labeled with the target program counter $p c$.

The general behavior of $\operatorname{Eager}_{k}(\mathcal{P}, p c)$ is as follows. 
Procedure main works as a driver program that calls each component program $P_{i}$ in turn at most once. After checking that $t_{0}, \ldots, t_{n}$ all contains valid indices of component programs (i.e., $1 \leq t_{i} \leq n$ holds for each $t_{i}$ ), for each component program $P_{i}, i=1, \ldots, n$, control variables $t$ and $c x$ are assigned respectively with the current component index (i.e., $i$ ) and the first context at which the current component is active if any (according to the scheduling of components given by $\left.t_{0}, \ldots, t_{n}\right)$. If there is no such context, the next component program is processed. Otherwise, the shared variables are consistently assigned with the initial values (given by init, if the current context is the first one, or by $g_{c x}$ otherwise), and thread ${ }_{i}^{e}$ is called.

Once called, a component program must run through all the contexts in which it is active and report if the target $p c$ is reached by setting goal to true. This is ensured by the control code inserted in each function.

Unless the variable terminate holds true, after each step of the original program, it is possible (nondeterministically) to call the function contextSwitch. In each such call, the computation proceeds by trying to make a context-switch. In case there are no more contexts in which the current program is active, the function sets terminate to true and returns. Otherwise, the shared variables are assigned with the guessed values for the next context where the current program is active and contextSwitch returns.

Observe that the if-statements checking the value of terminate in the control code ensure that in case terminate is set to true in contextSwitch, the control returns from all the calls stored in the call stack up to the main function. Since terminate is set to true only when there are no more contexts to run for the current component program, it is correct to interrupt the execution of the current component program and return to main so that the next component program (if any) can be executed.

Also, observe that the added "assume(F);" statement halts any computation that would have reached a return statement in each function thread ${ }_{i}^{e}$. Therefore, a call to any thread $e_{i}^{e}$ returns if and only if all the contexts in which $P_{i}$ is active have been successfully executed (i.e., terminate is set to true).

When all programs have successfully terminated we check the variable goal, and if it holds true, then the label Target is reached, else the program halts.

Note that if the guessed tuples $g_{1}, \ldots, g_{k}$ and $t_{0}, \ldots, t_{k}$ do not correspond to an actual computation of $\mathcal{P}$ then there will be at least one component program $P_{i}$, with $1 \leq i \leq n$, that cannot match the sequence $g_{1}, \ldots, g_{k}$ and thus the call to thread $i$ will not return.

From the above arguments, we conclude that a program counter $p c$ is reachable within $k$ context-switches in a computation of $\mathcal{P}$ if and only if Target in main is reachable in a computation of $\operatorname{Eager}_{k}(\mathcal{P}, p c)$. Therefore,

Theorem 1. Given an integer $k \geq 0$, a concurrent program $\mathcal{P}$ and a program counter $p c, p c$ is reachable in $\mathcal{P}$ within at most $k$ context-switches if and only if Target is reachable in Eager $_{k}(\mathcal{P}, p c)$. 


\section{Translation Scheme: The Lazy Approach}

We now detail the description of the translation of a concurrent program $\mathcal{P}$ under a context-switching bound $k$ to a sequential program using the lazy approach $\left(\right.$ denoted $\operatorname{Lazy}_{k}(\mathcal{P})$ ), and argue its correctness.

The structure of $\operatorname{Lazy}_{k}(\mathcal{P})$ is similar to that of $\operatorname{Eager}_{k}(\mathcal{P}, p c)$. We keep all the variables we used earlier except for $t$ and goal which are not needed here. Also, besides variable $c x$, we need a second global variable, $i c$, to store a context number (from 1 to $k$ ). Recall that for each context $c$ we need to run the component program $P_{i}$ that is active in $c$ through all the contexts $c^{\prime}$ in which $P_{i}$ was active, from the first context through context $c$. We use $c x$ to store the context $c$ and $i c$ to keep track of the context $c^{\prime}$ in the above computation.

In $\operatorname{Lazy}_{k}(\mathcal{P})$ each function $P$ of $\mathcal{P}$ is translated to a function $P^{l}$ which is similar $P^{e}$ in Section 4, except that here we do not need to set the global variable goal when the target program counter is reached. The remaining functions are main and contextSwitch, which are significantly different from those described in Section 4, and are given in Figure 4.

For each context $c x$, function main iteratively: (1) chooses the component program $P_{i}$ which is active in context $c x,(2)$ determines the first context $i c$ in which $P_{i}$ is active starting from context 0 through context $c x,(3)$ assigns the shared variables consistent with $i c$ (i.e., if $i c=0$ then the shared variables are assigned by function init, otherwise they are assigned with the values they have at the context-switch $i c$ ), and (4) calls function thread ${ }_{i}^{l}$.

In main, the call to function thread ${ }_{i}^{l}$ executes the component program $P_{i}^{l}$ through all the contexts in which it is active, from the first one up to $c x$. In fact,

Let $g$ : shared variables of $\mathcal{P} ; \quad$ Let $g_{1}, \ldots, g_{k}$ : be $k$ copies of $g$ decl int $c x, i c, t_{0}, \ldots, t_{k}$; decl bool terminate;
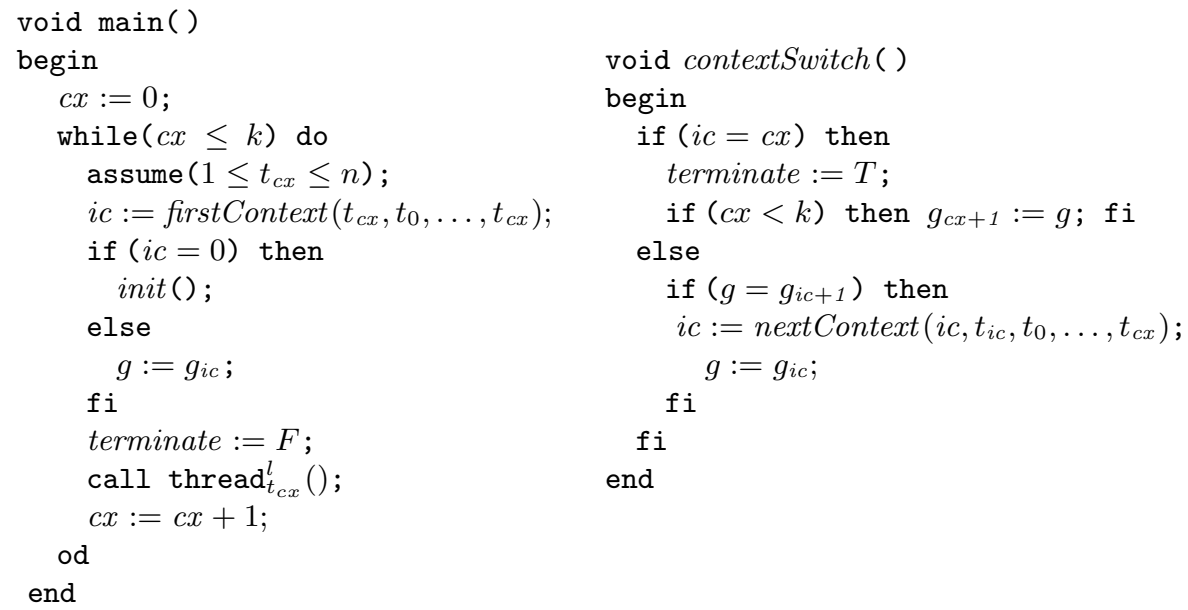

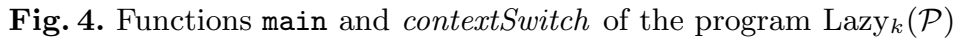


when thread ${ }_{i}^{l}$ is called the computation of $P_{i}^{l}$ starts at the beginning of context $i c$ (which is set to the first context where $P_{i}^{l}$ is active). After any step of $P_{i}^{l}$, it is possible to call contextSwitch (terminate is set to false before calling thread ${ }_{i}^{l}$ in function main) and then perform a context-switch if possible.

In function contextSwitch, if $i c<c x$, we check if it is possible to perform a context-switch by determining if the current value of the shared variables matches the stored value for the next context-switch. If so, the next context $c$ in which $P_{i}$ is active is computed and the shared variables are assigned with the value at the beginning of context $c$ (i.e., with $g_{c}$ ). Observe that since we know that $P_{i}$ is active in $c x$, there will always be such a context $c$.

From the last observation, we also have that if $i c<c x$ does not hold then $i c=c x$ must hold. Thus, in the remaining case, i.e. $i c=c x$, it is possible to make a context-switch at any point. This is correct, since we are executing $P_{i}$ in context $c x$, which is newly added before making this call to thread ${ }_{i}^{l}$ in main, and thus we have no requirements to match. When context-switching in this case, we only need to store the values of the shared variables in $g_{c x+1}$ (if we are not yet in the last context $k$ ) and flag that this call to thread ${ }_{i}^{l}$ has terminated by setting terminate to true.

From the above observations, any computation $\pi$ of $\mathcal{P}$ can be executed stepby-step by program $\operatorname{Lazy}_{k}(\mathcal{P})$, and thus if $\pi$ visits a program counter $p c$, the corresponding computation of $\operatorname{Lazy}_{k}(\mathcal{P})$ will also visit $p c$.

Now consider any computation $\pi$ of $\operatorname{Lazy}_{k}(\mathcal{P})$ which successfully terminates (i.e., the return statement of function main is reached). Recall that by construction each function thread ${ }_{i}^{l}$ has a statement "assume $(F)$ " guarding each return

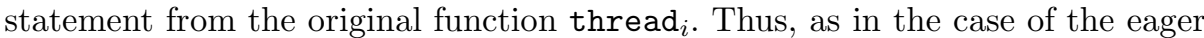
approach construction, the only way for a call to thread ${ }_{i}^{l}$ to return is by setting the variable terminate to true.

From all the above observations, all calls to each thread ${ }_{i}^{l}$ made from main return if and only if the computed sequence $g_{1}, \ldots, g_{k}$ is matched by a computation of $\mathcal{P}$. Therefore, for each computation $\pi$ of $\operatorname{Lazy}_{k}(\mathcal{P})$ which successfully terminates and which visits a program counter $p c$ of $\mathcal{P}$ there is a corresponding computation $\pi^{\prime}$ of $\mathcal{P}$ which visits $p c$. Hence,

Theorem 2. Given an integer $k \geq 0$, a concurrent program $\mathcal{P}$ and a program counter $p c, p c$ is reachable in $\mathcal{P}$ within at most $k$ context-switches if and only if pc is reachable in $\operatorname{Lazy}_{k}(\mathcal{P})$.

\section{Experiments}

Boolean programs. Boolean programs are programs where the data-domain is only Boolean. Since the data-domain is finite, Boolean programs can be subject to decidable model-checking for analyzing reachability. In particular, it is well known that sequential Boolean programs have a decidable reachability problem, and that for concurrent Boolean programs, reachability is undecidable (due to multiple stacks). However, it has also been shown that reachability problem for concurrent Boolean programs under a context-switching bound is decidable. 


\begin{tabular}{|c|c|c|c|c|c|c|c|c|c|c|c|}
\hline \multirow{2}{*}{$\begin{array}{l}\text { Context } \\
\text { switches }\end{array}$} & \multicolumn{2}{|c|}{ 1-adder, 1-stopper } & \multicolumn{3}{|c|}{ 2-adders, 1-stopper } & \multicolumn{3}{|c|}{ 1-adder, 2-stoppers } & \multicolumn{3}{|c|}{ 2-adders, 2-stoppers } \\
\hline & Eager & Lazy & & \begin{tabular}{|l|} 
Eager \\
\end{tabular} & $\overline{\text { Lazy }}$ & & Eager & $\overline{\text { Lazy }}$ & & Eager & Lazy \\
\hline 1 & $\overline{0.1}$ & $\overline{0.1}$ & $\mathrm{~N}$ & 0.2 & $\overline{0.1}$ & $\mathrm{~N}$ & 0.1 & 0.1 & $\mathrm{~N}$ & $\overline{0.2}$ & $\overline{0.1}$ \\
\hline 2 & 0.3 & 0.2 & $\mathrm{~N}$ & 0.9 & 0.8 & $\mathrm{~N}$ & 0.7 & 0.9 & $\mathrm{~N}$ & 1.6 & 2.0 \\
\hline 3 & 43.3 & 1.4 & $\mathrm{~N}$ & 135.9 & 6.3 & $\mathrm{Y}$ & 70.1 & 0.4 & $\mathrm{Y}$ & 177.6 & 0.8 \\
\hline 4 & 73.6 & 5.5 & $\mathrm{Y}$ & 1601.0 & 2.6 & $\mathrm{Y}$ & 597.2 & 2.9 & $\mathrm{Y}$ & out of mem. & 7.5 \\
\hline 5 & $\mathrm{~N} \mid 930.0$ & 20.2 & $\mathrm{Y}$ & - & 18.0 & $Y$ & - & 14.0 & $\mathrm{Y}$ & out of mem. & 66.5 \\
\hline 6 & $\mathrm{~N}$ & 66.8 & Y & & 122.9 & $Y$ & & 66.1 & $\mathrm{Y}$ & out of mem. & 535.9 \\
\hline
\end{tabular}

Fig. 5. Experimental results on the Bluetooth driver example. Times are expressed in seconds, "-" denotes timeout in 30 minutes and $\mathrm{Y} / \mathrm{N}$ denote if the target is reachable.

Translation for Boolean programs. We have implemented both the eager and lazy translations described in the previous sections for Boolean programs, in order to reduce the reachability of concurrent Boolean programs under a context-switching bound to the (decidable) problem of sequential Boolean program reachability. We then subject the sequential program to the reachability model-checker Moped 22. Our goal is to show the applicability of the translations, and in showing that, the lazy translation can outperform the eager one; we show this on a class of Bluetooth driver examples.

The first concurrent program we consider illustrates the difference between the eager and the lazy analysis; we consider the permutation example with 16-bits (a four-bit version of this program is shown in Figure 11). Initially, only thread ${ }_{1}$ can evolve as thread $_{2}$ is blocked on its first statement. thread sets all 16-bits to false and, before returning, sets test to true. Now, thread 2 can take over and goes into an infinite loop in which the bits are shuffled

\begin{tabular}{|c|r|r|r|}
\hline Context & \multicolumn{3}{|c|}{$\begin{array}{r}\text { permutation } \\
\text { Switches }\end{array}$} \\
\cline { 2 - 4 } & \multicolumn{1}{|c|}{ Eager } & Lazy \\
\hline 1 & $\mathrm{~N}$ & 6.97 & 0.1 \\
2 & $\mathrm{~N}$ & 194.7 & 1.0 \\
3 & $\mathrm{~N}$ & out of mem. & 122.5 \\
\hline
\end{tabular}

Table 1. Experiments of permutation program with 16 bits. Times are expressed in seconds producing sooner or later all permutations of the 16-bits. Since all the bits are set to false, only one permutation of the 16-bits is possible in the execution. The table with the experiments for permutation is reported in Table 1. As it is evident, the eager version simply fails after a few context-switches. In the eager approach all the global variables are guessed at the beginning and all the threads are executed on the guesses. Therefore this does not prevent thread 2 to work on arbitrary values of $x_{1}, \ldots, x_{16}$ and hence ends up exploring a large and complex state-space (storing all permutations requires large BDDs). The lazy version on the other hand only considers reachable states and hence works well on this example.

The second set of experiments is related to a concurrent boolean program modeling a Windows NT Bluetooth driver [11. Figure 5 depicts the experimental results. This driver has two types of threads: stoppers and adders. A stopper calls a stopping procedure to halt the driver, while an adder calls a procedure to perform $\mathrm{I} / \mathrm{O}$ in the driver. The $\mathrm{I} / \mathrm{O}$ is successfully handled if the driver is not stopped, and an error state is reached otherwise. The pending I/O requests to 
the driver are maintained in a counter which is modeled with five bits in our Boolean model of the program.

We have considered four different configurations, by considering one or two adders, and one or two stoppers. We translated the corresponding concurrent programs both with the lazy and eager version of our translator allowing up to six context-switches, and run Moped on them. As from Figure 5, the lazy scheme performs a lot better than the eager one, and the performance gap between them increases with the increase in the number of context-switches.

\section{$7 \quad$ Future Directions}

There are two interesting future directions we see. One is to see whether using the lazy translation scheme presented here in the context of deductive verification of concurrent $\mathrm{C}$ programs as done in [5] leads to more efficient analysis. Second, it would be interesting to see whether the scheme proposed here can be extended to more general concurrent programs with dynamic and unbounded thread creation.

\section{References}

1. Bouajjani, A., Fratani, S., Qadeer, S.: Context-bounded analysis of multithreaded programs with dynamic linked structures. In: Damm, W., Hermanns, H. (eds.) CAV 2007. LNCS, vol. 4590, pp. 207-220. Springer, Heidelberg (2007)

2. Esparza, J., Schwoon, S.: A BDD-based model checker for recursive programs. In: Berry, G., Comon, H., Finkel, A. (eds.) CAV 2001. LNCS, vol. 2102, pp. 324-336. Springer, Heidelberg (2001)

3. La Torre, S., Madhusudan, P., Parlato, G.: Context-bounded analysis of concurrent queue systems. In: Ramakrishnan, C.R., Rehof, J. (eds.) TACAS 2008. LNCS, vol. 4963, pp. 299-314. Springer, Heidelberg (2008)

4. La Torre, S., Madhusudan, P., Parlato, G.: Analyzing recursive programs using a fixed-point calculus. In: PLDI (2009)

5. Lahiri, S.K., Qadeer, S., Rakamarić, Z.: Static and Precise Detection of Concurrency Errors in Systems Code using SMT solvers. In: Bouajjani, A., Maler, O. (eds.) CAV 2009. LNCS, vol. 5643, pp. 509-524. Springer, Heidelberg (2009)

6. Lal, A., Reps, T.W.: Reducing concurrent analysis under a context bound to sequential analysis. In: Gupta, A., Malik, S. (eds.) CAV 2008. LNCS, vol. 5123, pp. 37-51. Springer, Heidelberg (2008)

7. Lal, A., Touili, T., Kidd, N., Reps, T.W.: Interprocedural analysis of concurrent programs under a context bound. In: Ramakrishnan, C.R., Rehof, J. (eds.) TACAS 2008. LNCS, vol. 4963, pp. 282-298. Springer, Heidelberg (2008)

8. Musuvathi, M., Qadeer, S.: Chess: Systematic stress testing of concurrent software. In: Puebla, G. (ed.) LOPSTR 2006. LNCS, vol. 4407, pp. 15-16. Springer, Heidelberg (2007)

9. Musuvathi, M., Qadeer, S.: Iterative context bounding for systematic testing of multithreaded programs. In: PLDI, pp. 446-455. ACM, New York (2007)

10. Qadeer, S., Rehof, J.: Context-bounded model checking of concurrent software. In: Halbwachs, N., Zuck, L.D. (eds.) TACAS 2005. LNCS, vol. 3440, pp. 93-107. Springer, Heidelberg (2005) 
11. Qadeer, S., Wu, D.: Kiss: keep it simple and sequential. In: PLDI, pp. 14-24. ACM, New York (2004)

12. Ramalingam, G.: Context-sensitive synchronization-sensitive analysis is undecidable. ACM Trans. Program. Lang. Syst. 22(2), 416-430 (2000)

13. Suwimonteerabuth, D., Esparza, J., Schwoon, S.: Symbolic context-bounded analysis of multithreaded java programs. In: Havelund, K., Majumdar, R., Palsberg, J. (eds.) SPIN 2008. LNCS, vol. 5156, pp. 270-287. Springer, Heidelberg (2008) 